\title{
Loron / Santa Marina - Busuja (Tar-Vabriga, Poreč, Croatie)
}

Campagne de fouilles 2013

Corinne Rousse, Vladimir Kovačić, Solenn De Larminat, Valerio De Leonardis, Paola Maggi, Claudio Taffetani et Fabrizio Terrizzi

\section{(2) OpenEdition Journals}

Édition électronique

URL : http://journals.openedition.org/cefr/1212

DOI : $10.4000 /$ cefr. 1212

ISSN : 2282-5703

Éditeur

École française de Rome

Référence électronique

Corinne Rousse, Vladimir Kovačić, Solenn De Larminat, Valerio De Leonardis, Paola Maggi, Claudio Taffetani et Fabrizio Terrizzi, « Loron / Santa Marina - Busuja (Tar-Vabriga, Poreč, Croatie) », Chronique des activités archéologiques de l'École française de Rome [En ligne], Balkans, mis en ligne le 12 juin 2014, consulté le 07 août 2019. URL : http://journals.openedition.org/cefr/1212 ; DOI : 10.4000/cefr.1212

Ce document a été généré automatiquement le 7 août 2019

(c) École française de Rome 


\title{
Loron / Santa Marina - Busuja (Tar- Vabriga, Poreč, Croatie)
}

Campagne de fouilles 2013

\author{
Corinne Rousse, Vladimir Kovačić, Solenn De Larminat, Valerio De \\ Leonardis, Paola Maggi, Claudio Taffetani et Fabrizio Terrizzi
}

\section{NOTE DE L'AUTEUR}

Sous la direction de Vladimir Kovačić (Zavičajni muzej Poreštine) et Corinne Rousse (AixMarseille université, UMR 7299 Centre Camille Jullian), ont collaboré aux opérations de terrain : Valerio De Leonardis (doctorant, Universiteit van Amsterdam, Università degli studi di Roma Tre), Perrine Kossmann (Université de Bourgogne, UMR 6298 ARTEHIS) et Claudio Taffetani (doctorant, Aix-Marseille Université, UMR 7299 Centre Camille Jullian, Università degli studi di Roma Tre) - chefs de secteur-; Nina Gostinski, Željka Grgurić, Dora Simic (master I, Sveučilišta u Zagrebu / Université de Zagreb), Tina Kompare (doctorante, Univerza na Primorskem / Université de Primorska), Nabil Hamzaoui (doctorant, Université de Toulouse), Charline Legras (master I, Aix Marseille Université), Caterina Pane (master II, Università degli studi di Roma « La Sapienza »), Fabrizio Terrizzi (master II, Università degli studi di Roma « Tor Vergata ») et Chloé Vachia (doctorante, Université de Tours).

Claudio Taffetani est l'auteur des planimétries et relevés topographiques. Valerio De Leonardis a conduit les opérations de dégagement et d'étude de la citerne (juin 2013). Solenn De Larminat (post-doctorante, Centre « Antiquité classique et tardive », UMR 8167 Orient et Méditerranée) a réalisé l'étude archéo-anthropologique de la sépulture médiévale et Fabrizio Terrizzi le catalogue des monnaies déposées avec le défunt. Comme chaque année, Paola Maggi (Università degli studi di Trieste) a coordonné l'inventaire et l'étude du mobilier. Une première évaluation archéo-botanique a également été conduite par Nuria Rovira (UMR 5140 Archéologie des sociétés méditerranéennes) dans le cadre des recherches paléo-environnementales menées avec la mission franco-croate de Busuja 
(sous la direction de M.-B. Carre), au sein d'un programme commun (ISTRIE) soutenu par le ministère des affaires étrangères.

1 Le site de Loron, sur la commune de Tar-Vabriga, correspond à un grand domaine maritime englobant la totalité du promontoire de Loron et les baies voisines de Santa Marina au nord et de Červar Porat au sud (fig. 1).

2 L'épigraphie, principalement sur les timbres d'amphores à huile Dressel 6B, permet de reconstituer l'histoire de cette prestigieuse propriété sénatoriale, fondée vers 10 ap. J.-C. par Sisenna Statilius Taurus, fils cadet du grand Statilius Taurus, ami d'Auguste, puis propriété de Messaline, Calvia Crispinilla et à partir de Domitien, des empereurs. L'histoire se perd avec Hadrien, dernier propriétaire attesté par les timbres, mais les données archéologiques montrent une continuité d'occupation jusque dans la deuxième moitié du V $\mathrm{V}^{\mathrm{e}}$ s. ap. J.-C.

Depuis 2012, les recherches franco-croates se concentrent sur la caractérisation des secteurs résidentiels repérés sur le promontoire (2012-2016), parallèlement au complexe artisanal déjà étudié (1994-2011). L'objectif est de préciser l'organisation de ce domaine aristocratique, en relation avec l'environnement littoral et les grandes propriétés voisines. Soutenu par l'École française de Rome et le Ministère des Affaires étrangères, ce programme associe étroitement la Surintendance d'Istrie et le Musée territorial du Parentin (Zavičajni muzej Poreštine) pour les partenaires croates, le Centre Camille Jullian (UMR 7299) et l'Université d'Aix-Marseille pour la partie française. Le Centre Ausonius-Université de Bordeaux III (UMR 5607), le CEREGE (UMR 7330), le GET (UMR 5563) et le Laboratoire Archéologie des sociétés méditerranéennes (UMR 5140) collaborent également à la mission pour les études sur le mobilier, les prospections géophysiques et les analyses paléo-environnementales. Enfin, la commune de Tar-Vabriga apporte un soutien logistique et financier à nos recherches.

4 En 2013, les opérations de terrain (3-12 juin ; 25 juillet-3 août) se sont déployées sur deux secteurs d'habitat ciblés par les précédentes prospections, distants l'un de l'autre de $400 \mathrm{~m}$ environ. Le premier correspond au module occidental du complexe artisanal de Loron, sur la rive sud du promontoire, dans ce qu'on suppose être un quartier résidentiel lié à l'atelier et destiné au personnel, esclaves ou libres, travaillant dans les fours (fig. 1 : Loron). Les sondages réalisés pour mieux caractériser l'occupation antique ont aussi révélé, pour la première fois sur le site, un contexte médiéval, avec la découverte d'une sépulture masculine datée de la fin du XIII ${ }^{\mathrm{e}} \mathrm{s}$. par un petit trésor monétaire.

5 Le second secteur étudié se situe sur la rive nord du promontoire, dans les reliefs boisés qui surplombent la baie de Santa Marina, au niveau de l'emprise supposée de la résidence aristocratique et impériale (fig. 1 : Santa Marina). Il s'agit de la première intervention de fouille dans cette zone, suite à des campagnes de prospections géophysiques et terrestres réalisées en 2011-2012. Notre intervention s'est concentrée sur une imposante citerne conservée encore en élévation, qui a pu être pour la première fois dégagée et étudiée jusqu'au pavement. Suite à ces opérations, deux missions post-fouilles (15 juillet-3 août ; 18-22 novembre) ont été conduites pour traiter le mobilier et compléter l'étude de la sépulture. 
Fig. 1 - Vue d'ensemble du promontoire de Loron, avec le complexe artisanal de Loron et les vestiges antiques du secteur de Santa Marina.

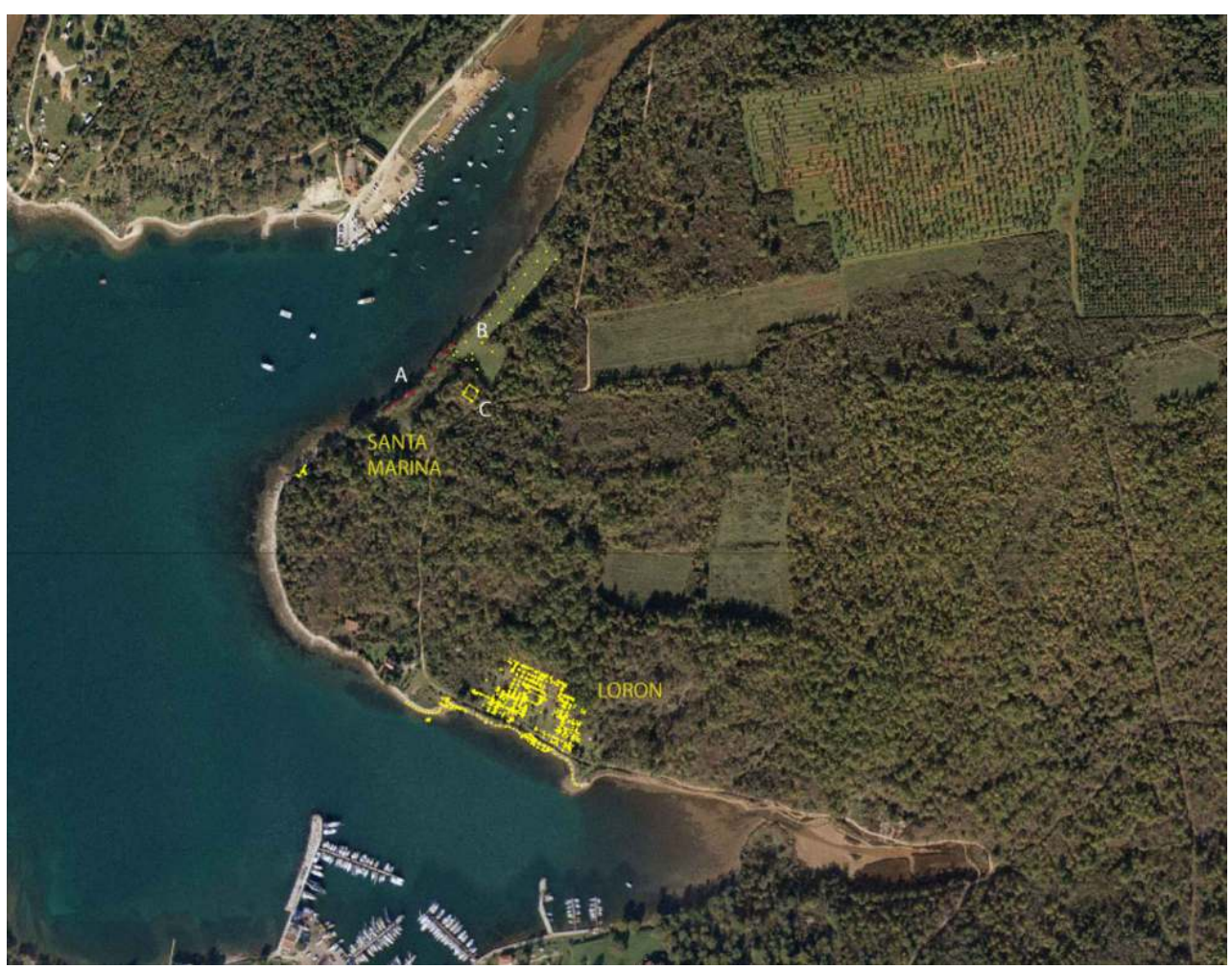

CAO C. Taffetani.

\section{Le secteur B du module occidental de Loron : sondages dans l'habitat associé au complexe artisanal}

L'organisation en deux modules du complexe artisanal de Loron est bien connue depuis les premières fouilles (Loron I) avec, de part et d'autre d'une large voie nord-sud descendant vers la plage, un module oriental dédié à la production des amphores et un module occidental caractérisé par la présence de petits thermes, probablement destinés au personnel et aux potiers travaillant dans l'atelier (fig. 2). Ce module occidental se présente sous la forme d'un long édifice quadrangulaire ( $65 \mathrm{~m}$ de long sur $25 \mathrm{~m}$ de large) comprenant, outre les bains, une série de petites pièces donnant sur la plage et en retrait, un vaste espace rectangulaire $(23 \times 45 \mathrm{~m})$, appelé espace 50 , dont il n'est pas encore possible de préciser l'architecture et la fonction (bâtiment couvert ou cour) ${ }^{1}$. L'opération réalisée en 2013 a consisté en sondages d'évaluation réalisés en particulier dans l'angle nord-est de l'espace 50. 
Fig. 2 - Plan d'ensemble du complexe artisanal de Loron : module oriental (atelier d'amphores) et module occidental.

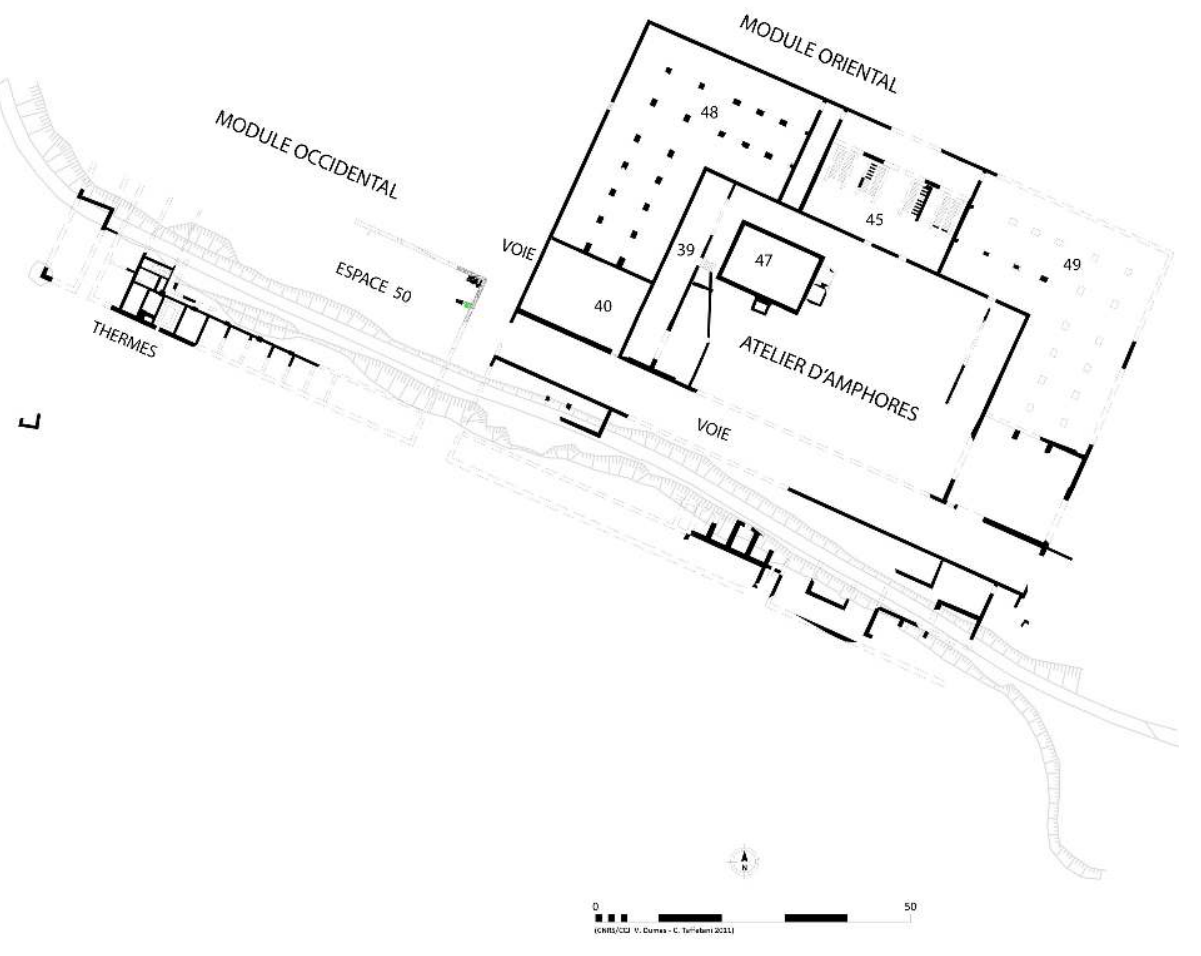

CAO C. Taffetani.

\section{L'espace 50 : les niveaux antiques}

7 Le sondage réalisé dans l'angle nord-est de l'espace $50(3 \times 6 \mathrm{~m})$ confirme que l'aménagement de cet espace remonte à la phase originale du complexe (vers 10 ap. J.-C.). Des éléments de pavement d'époque tardive ( $\mathrm{IV}^{\mathrm{e}}-\mathrm{V}^{\mathrm{e}} \mathrm{s}$.) retrouvés in situ attestent qu'un même niveau de circulation a été utilisé durant toute la période de fonctionnement de l'édifice, ce qui confirme les hypothèses de restitution déjà avancées pour le module oriental (atelier) où les plans de circulation ne sont pas conservés. Ce pavement, constitué de petites dalles posées à plat s'appuie sur la fondation des murs, le substrat géologique et un petit mur de terrasse destiné à contrecarrer la pente du terrain (fig. 3). Il est recouvert par une épaisse couche de destruction correspondant à la phase d'abandon et d'arasement du site, datée de la deuxième moitié du V $\mathrm{e}$ s. ap. J.-C.

8 Fig. 3 - Loron. Vue d'ensemble du sondage réalisé dans l'angle nord-est de l'espace 50 au niveau du substrat géologique. 


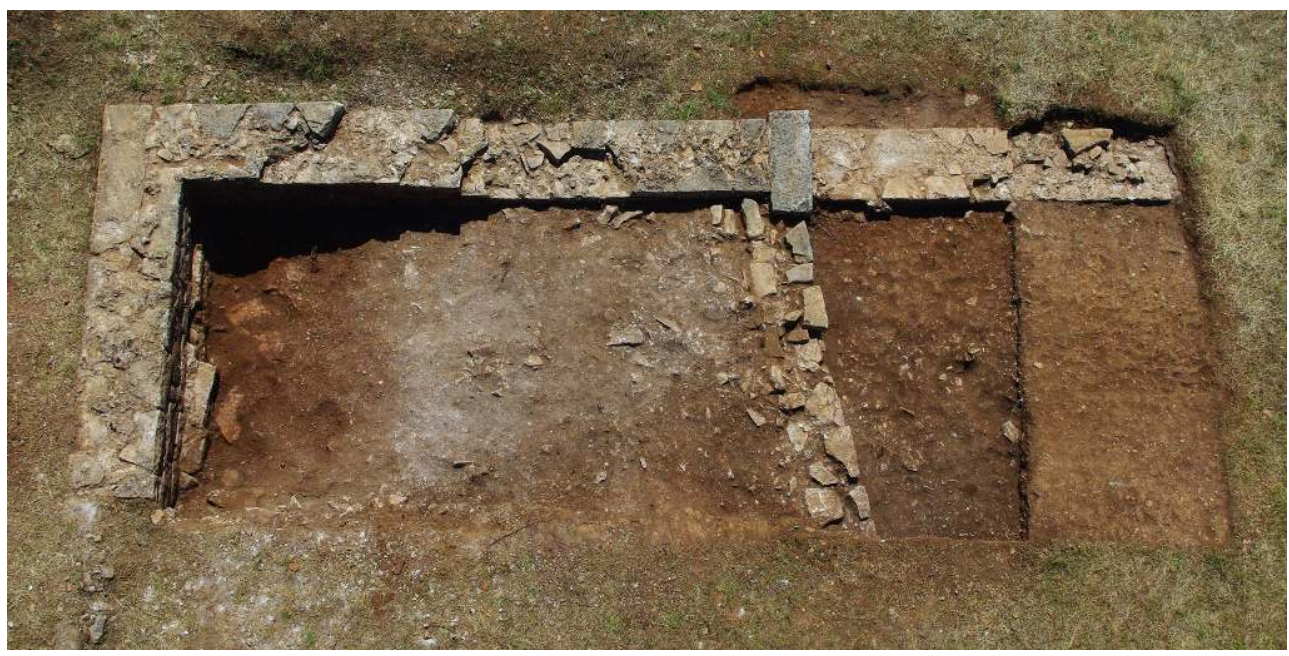

Cl. C. Taffetani.

\section{La sépulture médiévale et son petit trésor monétaire (fin $\mathrm{XIII}$ s.)}

Dans cette couche de destruction a été découverte à un niveau très superficiel une sépulture constituée par une simple fosse creusée le long du mur nord de l'espace 50, dans l'angle nord-est du bâtiment (fig. 4). Le défunt a été déposé allongé sur le dos, tête à l'ouest, sans autre mobilier funéraire qu'un lot de 9 deniers d'argent, découverts assemblés au niveau du bassin (fig. 5). Ce petit trésor suggère la présence d'une bourse enterrée avec le défunt. Les neufs monnaies d'argent correspondent à des deniers émis par les ateliers d'Aquilée et de Trieste, entre 1234-1254 (LOR 7014.7; LOR7014.8) et 1260-1282 (LOR7014.9). Leur excellent état de conservation a permis d'en dresser le catalogue complet, réalisé durant la campagne de terrain par Fabrizio Terrizzi (infra et fig. 6). L'arc chronologique resserré de ce trésor monétaire permet de proposer une datation de la sépulture dans les dernières décennies du XIII ${ }^{\mathrm{e}}$ s., ou plus tard au début XIV es. 
Fig. 4 - Loron. La sépulture médiévale SP 7006.

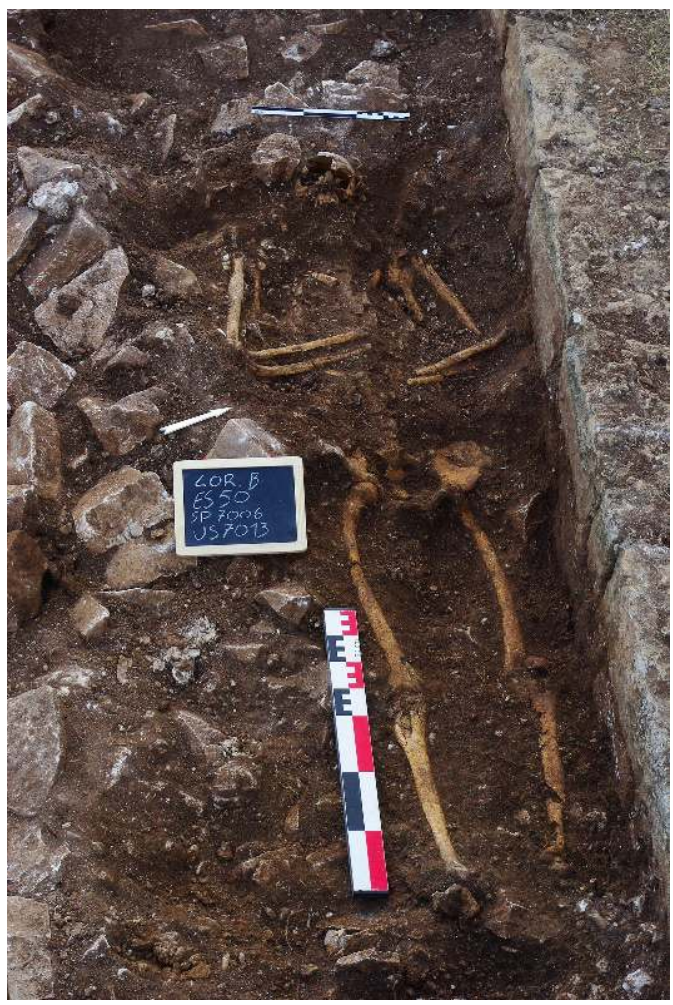

Cl. C. Rousse.

Fig. 5 - Loron. Planimétrie de la sépulture SP 7006 avec le trésor monétaire.

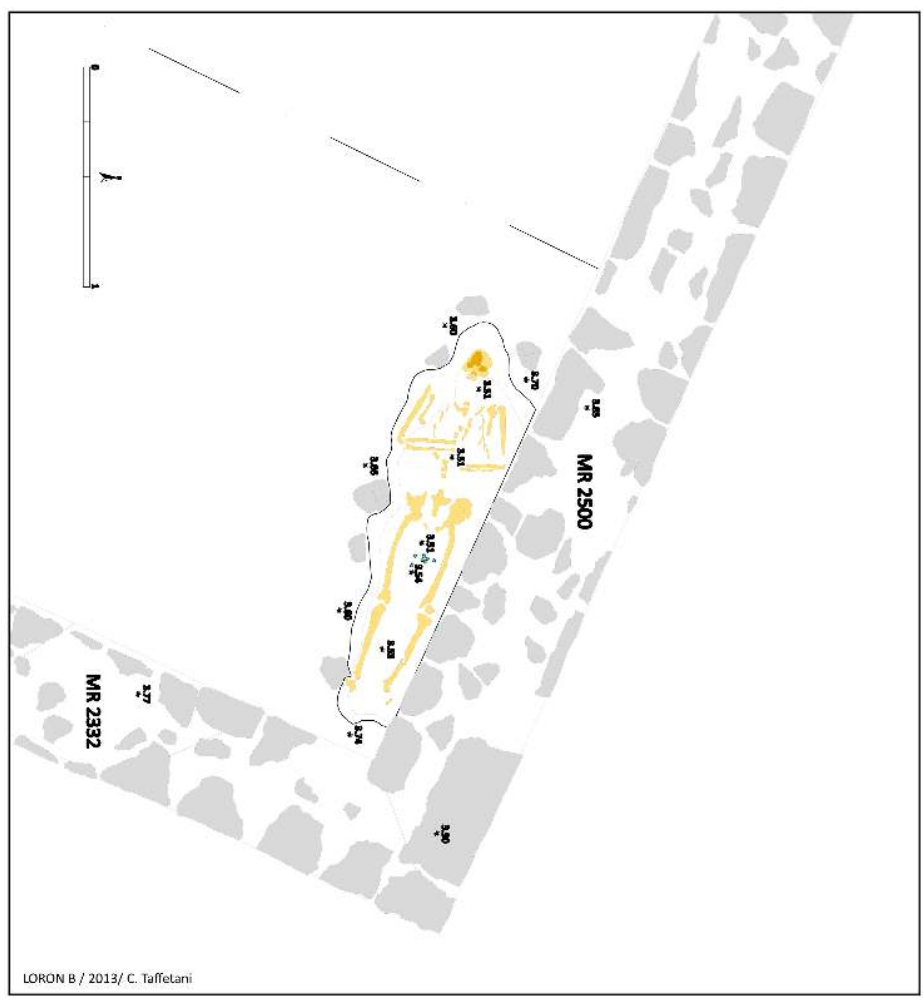

C. Taffetani. 
Annexe 1 : Liste des monnaies

(d'après l'inventaire complet réalisé par F. Terrizzi)

LOR7014.1 : Aquileia. Patriarca Gregorio di Montelongo. Denaro scodellato (Ar). 1251-1269

(fig. 6).

LOR7014.2: Aquileia. Patriarca Gregorio di Montelongo. Denaro scodellato (Ar). 1251-1269.

LOR7014.3: Aquileia. Patriarca Gregorio di Montelongo. Denaro scodellato (Ar). 1251-1269.

LOR7014.4: Aquileia. Patriarca Gregorio di Montelongo. Denaro scodellato (Ar). 1251-1269.

LOR7014.5: Aquileia. Patriarca Gregorio di Montelongo. Denaro scodellato (Ar). 1251-1269. (fig. 6).

LOR7014.6: Aquileia. Patriarca Gregorio di Montelongo. Denaro scodellato (Ar). 1251-1269.

LOR7014.7 : Trieste. Vescovo Volrico od Ulrico de Portis. Denaro (Ar). 1234-1254 (fig. 6).

LOR7014.8 : Trieste. Vescovo Volrico od Ulrico de Portis. Denaro (Ar). 1234-1254.

LOR7014.9 : Trieste. Vescovo Arlongo de' Visgoni. Denaro (Ar). 1260-1282 (fig. 6).

Fig. 6 - Loron. Monnaies provenant de la sépulture SP 7006.

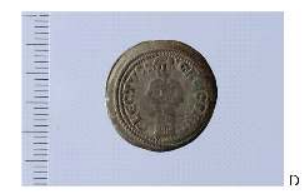

LOR7014.1

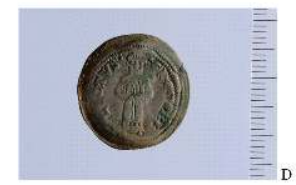

LOR7014.5

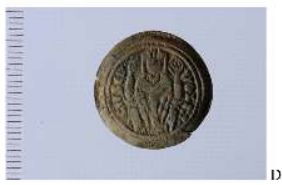

LOR7014.7

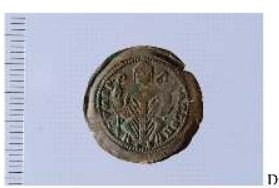

LOR7014.9
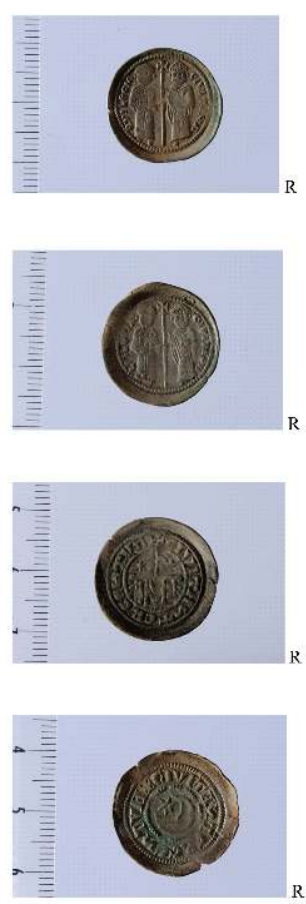

CL. L. DAMMELET, CCJ.

Premier contexte post-antique découvert sur le site, cette sépulture vient nuancer l'hypothèse d'un arasement général du site dès la fin de l'Antiquité, puisque elle est installée en correspondance étroite avec l'angle nord est de l'édifice antique : une partie des murs, certainement en ruine, devait être encore visible. Il devient aussi nécessaire de vérifier s'il s'agit d'un contexte funéraire isolé (sépulture d'urgence) en lien avec la 
fréquentation du promontoire dans le cadre d'un petit commerce régional (ressources de la mer, exploitation des ruines et du substrat comme carrière) ou d'une trace de l'occupation médiévale du promontoire, qui reste pour l'heure très mal documentée ${ }^{2}$. Le petit trésor associé au défunt représente en tout cas une somme qui n'est pas négligeable: si l'on compare avec d'autres trouvailles monétaires associées à des sépultures isolées d'époque médiévale en Italie ${ }^{3}$, le dépôt de Loron apparaitt comme l'un des plus riches.

\section{Annexe 2 : Étude archéo-anthropologique de la sépulture}

\section{(S. De Larminat)}

12 Le squelette de la sépulture primaire à inhumation SP 7006 est bien conservé. Les os sont généralement entiers (quelques extrémités diaphysaires, côtes et vertèbres fragmentés). Seules quelques phalanges de pieds sont absentes. L'individu est un adulte de plus de 30 ans d'après la synostose complète du squelette et l'aspect de la surface sacro-pelvienne iliaque $^{4}$. La méthode de diagnose probabiliste du sexe n'est pas déterminante en revanche, l'observation scopique du bassin indique que l'individu était un homme ${ }^{5}$. Il était de grande taille $(173,9 \pm 4,5 \mathrm{~cm})^{6}$. Le défunt souffrait du dos et du cou (tassements des lombaires et réactions ostéophytiques au niveau de l'ensemble des vertèbres et tout particulièrement sur les lombaires et les cervicales). Il présente, outre une densité importante de tartre autour des dents inférieures, une perte ante-mortem de 9 dents (essentiellement des molaires) et une usure occlusale importante, des abcès dentaires fortement développés au niveau des racines de la seconde prémolaire et des trois molaires inférieures gauches. Ces abcès ont provoqué une réaction inflammatoire du périoste latérale gauche de la mandibule. Ces infections buccales peuvent être une des causes de la mort de l'individu.

13 Le défunt a été déposé sur le dos, très légèrement sur le côté gauche, la tête à l'ouest, le regard vers le haut, dans une fosse oblongue présentant des bords inclinés réduisant la longueur de la fosse de $2 \mathrm{~m}$ à 1,65 $\mathrm{m}$ à son niveau inférieur. Le squelette épouse les formes de la fosse : le bloc crânio-facial, les pieds et les humérus sont ainsi surélevés par rapport au reste du corps. L'humérus droit se présente le long du corps alors que le gauche est légèrement éloigné. Les avant-bras sont ramenés sur la moitié supérieure du corps sans toutefois être croisés, de sorte que la main gauche se situait sur le ventre et la droite sur la poitrine gauche. Les membres inférieurs sont en extension sans que les genoux et les chevilles soient rapprochés. Les déplacements osseux, peu importants à l'exception de la mandibule située à 6-7 cm en contrebas du bloc crânio-facial, sont dus à des mouvements dans les espaces laissés libres lors de la disparition des chairs et lors de l'effondrement de la cage thoracique. D'après ces observations, le corps ne semble pas avoir été placé dans un contenant en bois non cloué, mais bien directement dans la fosse sans que celle-ci soit aménagée. Si le corps a été enveloppé dans un linceul, celui-ci n’était pas compressif. Une inhumation habillée peut alors être envisagée.

\section{La citerne de Santa Marina : une grande structure hydraulique d'époque romaine}

$\mathrm{Au}$ nord du promontoire, le long de la baie de Santa Marina et à $400 \mathrm{~m}$ environ du complexe artisanal, une concentration de vestiges indique la présence d'un secteur 
résidentiel encore à étudier. ${ }^{7}$ Sur la plage, des murs antiques perpendiculaires au rivage sont encore visibles (fig. 1, A). D'autres structures construites, dont un mur long de 100 mètres parallèle à la côte, ont été repérées par des prospections géophysiques (fig. 1, B). Mais c'est surtout la présence d'une vaste citerne encore en élévation qui confirme l'existence d'un habitat dans ce secteur : installée à flanc de pente et surplombant la baie (fig. 7), elle se distingue par des dimensions notables $(12,80 \times 13,60 \mathrm{~m})$ et une architecture particulièrement robuste qui explique la conservation des murs (fig. 8). Réalisée dans des conditions difficiles (déboisement, nettoyage et sondage manuels, suivie d'une nouvelle opération en juillet à la pelle mécanique), l'étude a permis de documenter précisément les différentes particularités architecturales du monument. Chaque paroi est ainsi constituée de deux murs parementés enserrant un noyau en béton hydraulique, qui constitue au final l'essentiel de l'élévation conservée (fig. 9). La présence d'un enduit hydraulique soigneusement lissé a également été observée par sondage dans la partie basse de la paroi interne de la citerne (fig. 10). Le pavement est en opus spicatum et appuyé directement sur le substrat (fig. 11). Il est situé à $3 \mathrm{~m}$ de profondeur par rapport au sommet conservé de l'élévation. Le sondage réalisé dans l'angle nord-est de la citerne montre que la structure a fait l'objet de spoliation, sans pouvoir préciser pour l'instant la durée d'utilisation de cette structure et la phase de comblement ou d'abandon. Une nouvelle intervention réalisée en juillet à la pelle mécanique (enlèvement des remblais superficiel) permet d'affirmer que cette citerne était couverte, avec un puits doté d'une imposante margelle $(2 \times 2 \times 0,7 \mathrm{~m})$ retrouvée in situ (fig. 12).

Fig. 7 - Loron. Vue de la citerne, en cours de dégagement.

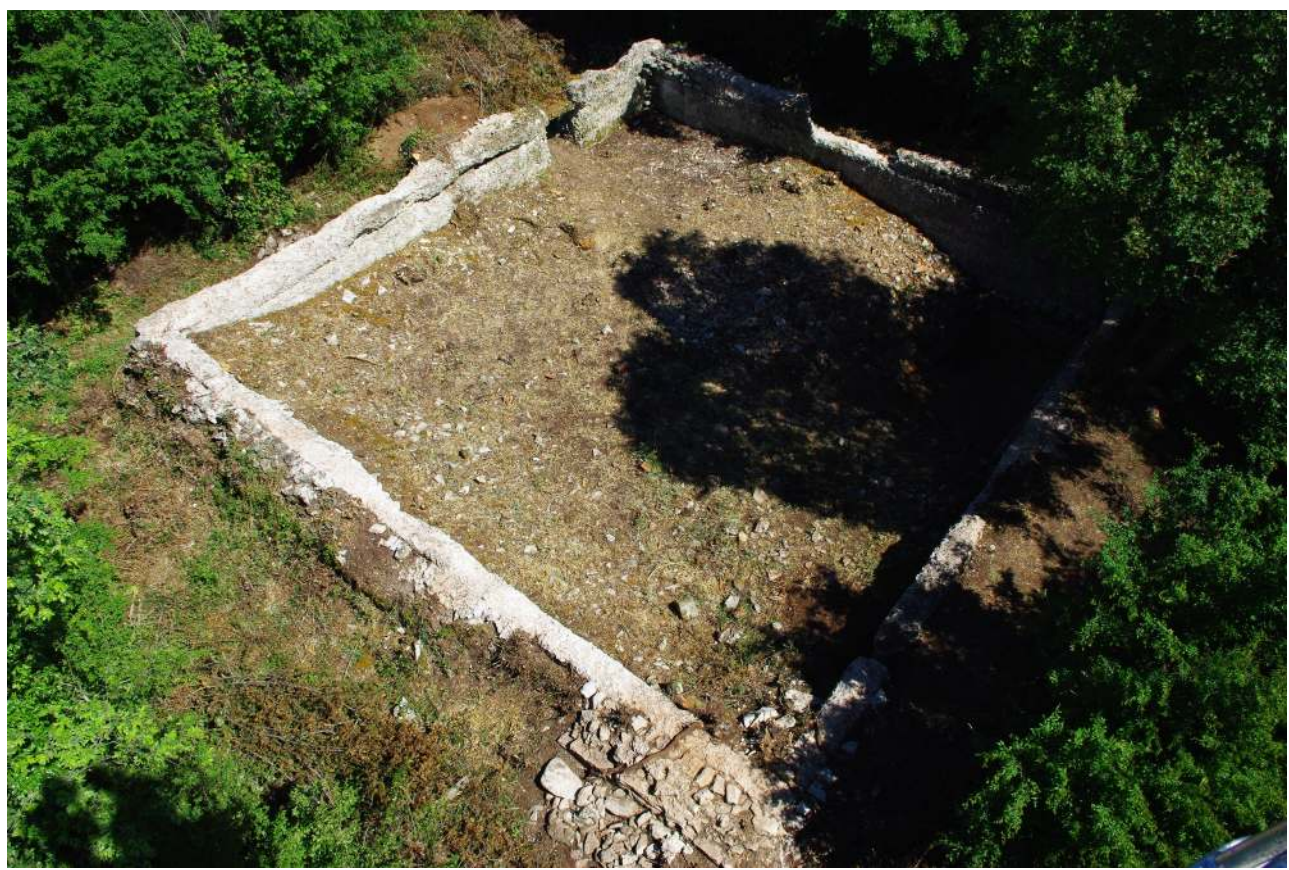

Cl. V. De Leonardis. 
Fig. 8 - Loron. Planimétrie de la citerne - état 2013.

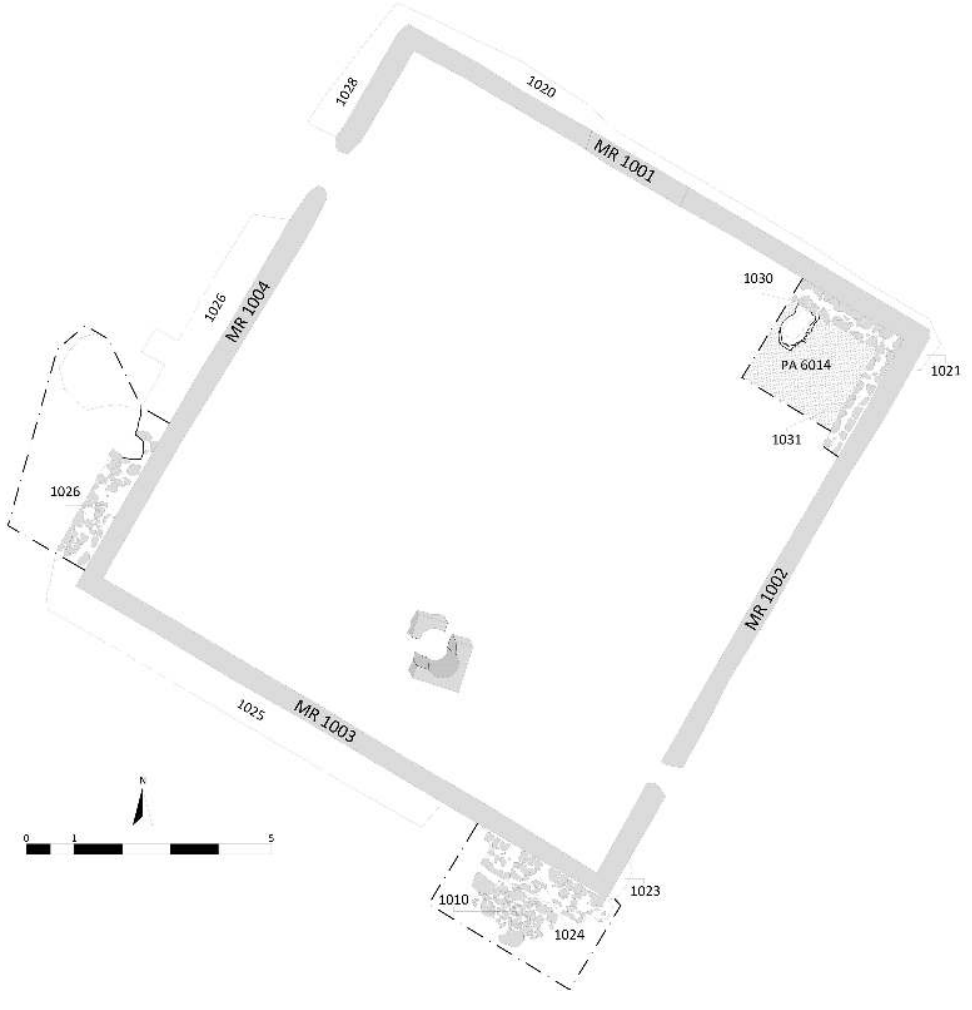

C. Taffetani.

Fig. 9 - Loron. Élévation conservée du mur nord - noyau en béton hydraulique.

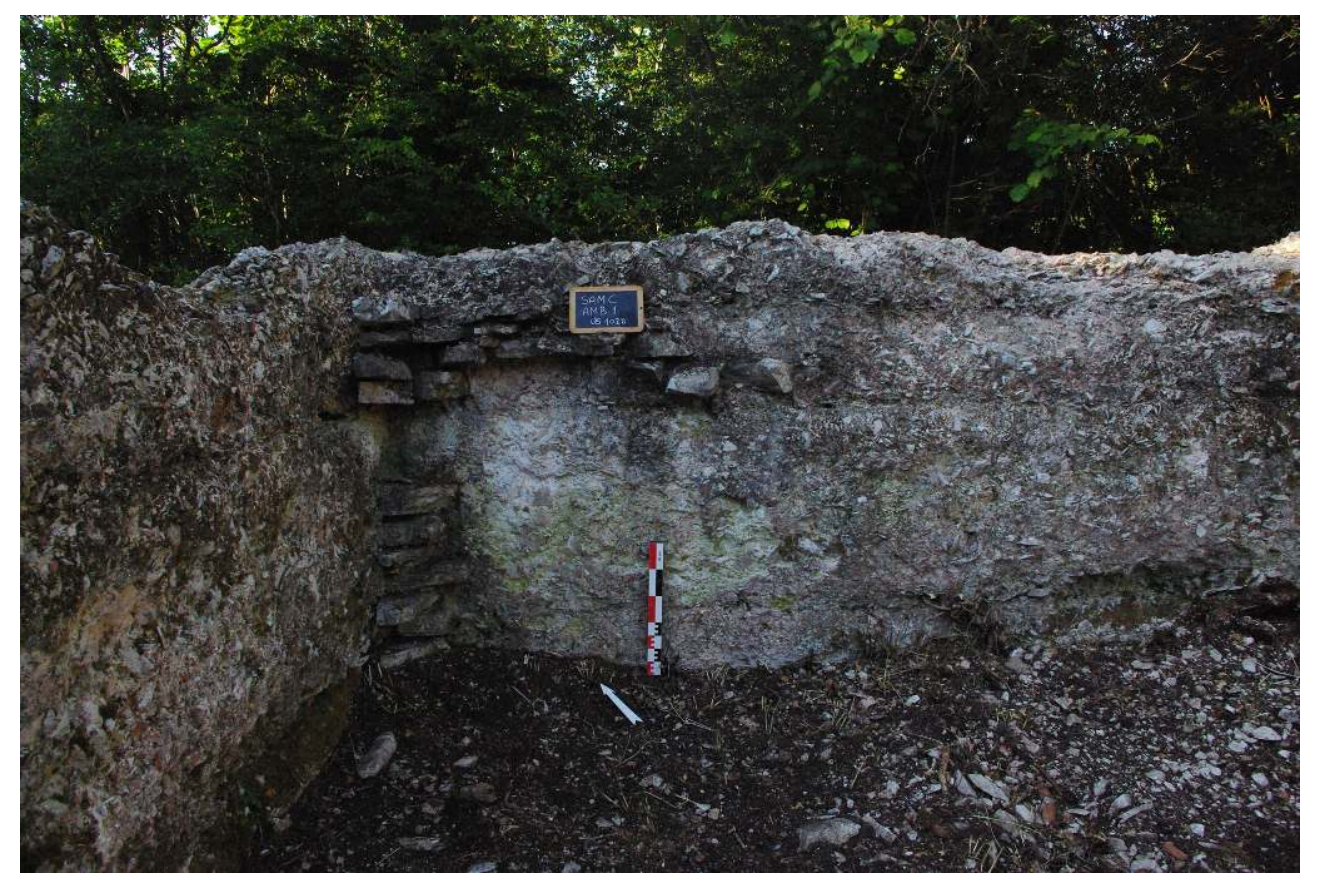

Cl. C. Rousse. 
Fig. 10 - Loron. Vue d'ensemble du sondage S1 réalisé dans l'angle nord-est de la citerne.

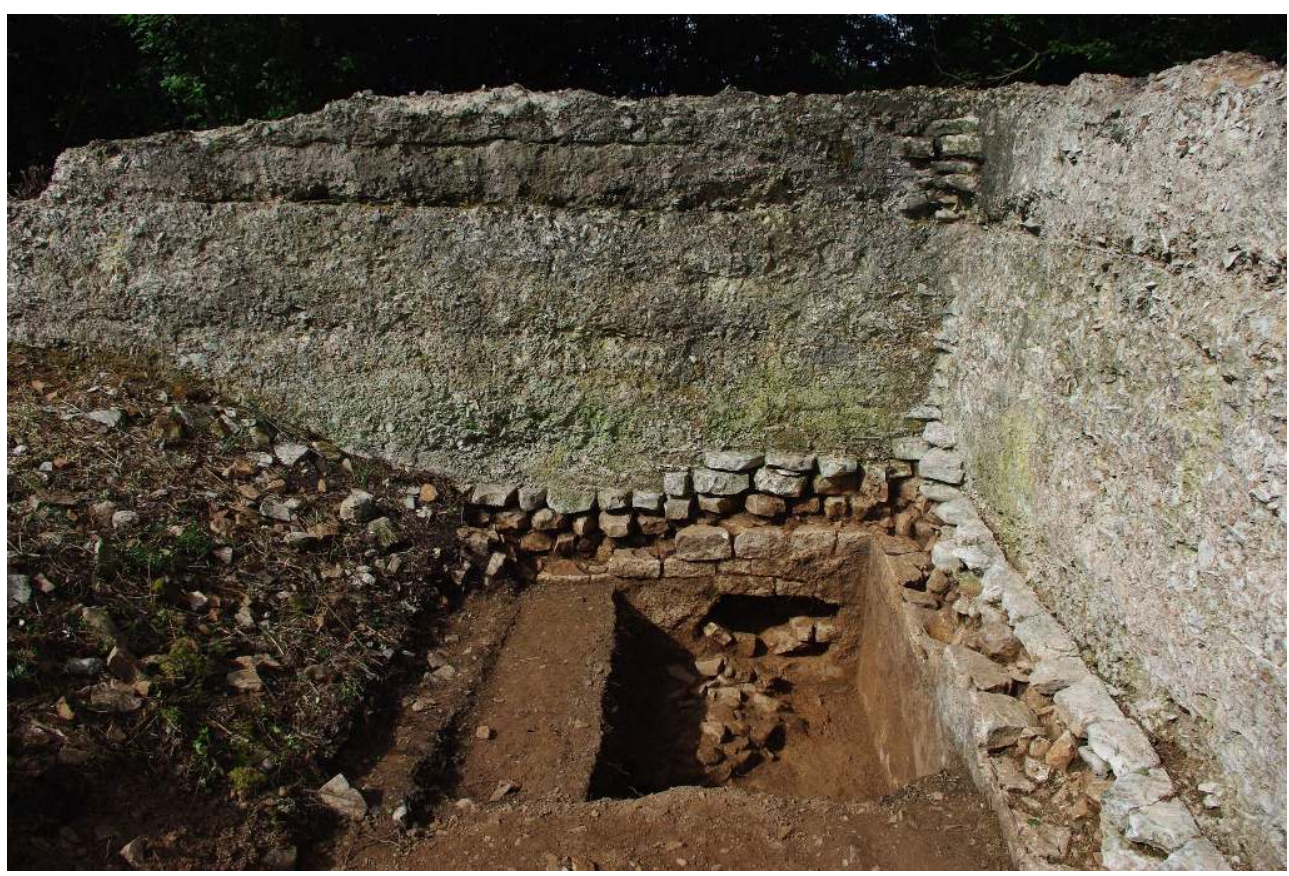

Cl. V. De Leonardis.

Fig. 11 - Loron. Détail du pavement et des parois internes de la citerne, avec trace de spoliation sondage S1.

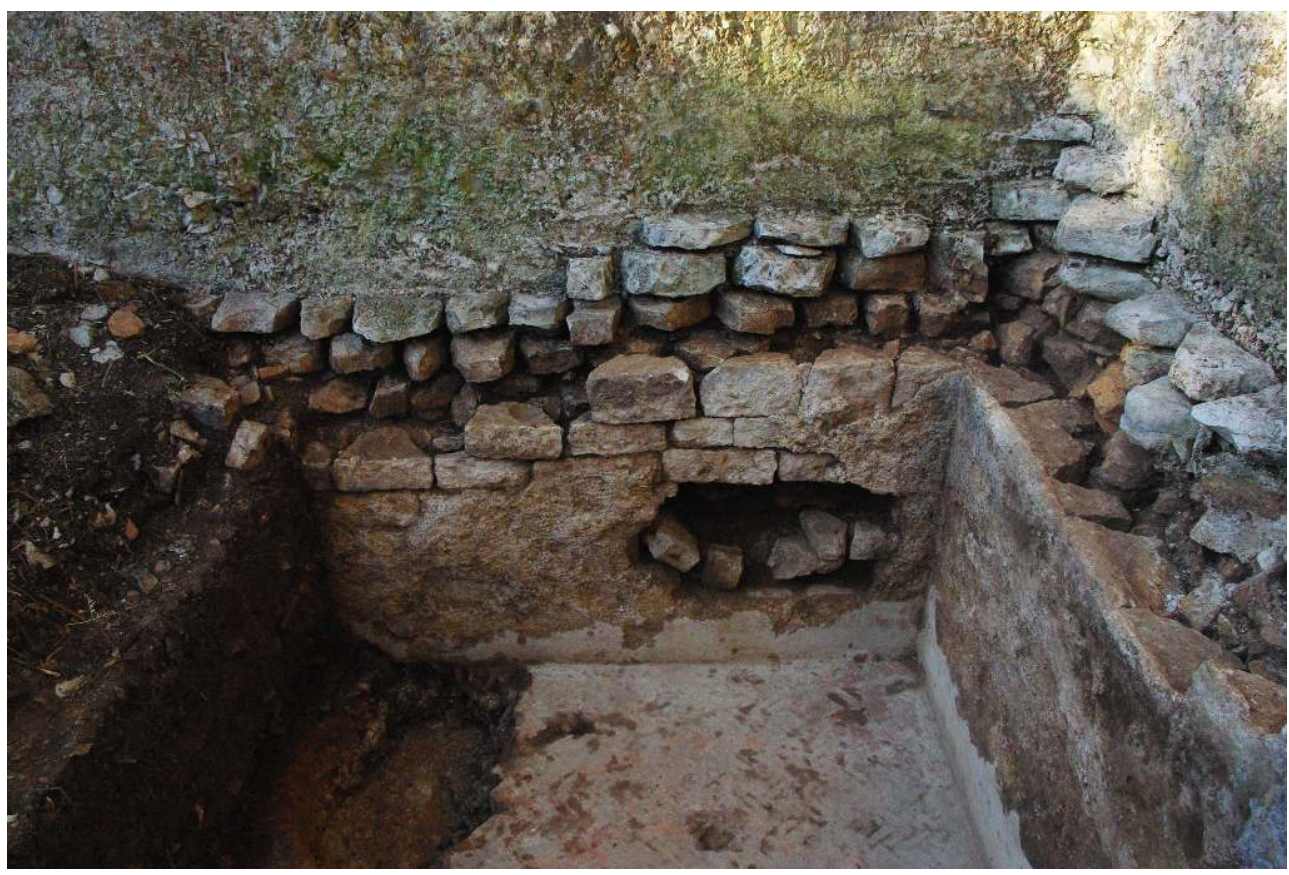

Cl. C. Rousse. 
Fig. 12 - Loron. Élément d'une margelle de puits découvert dans le niveau supérieur du comblement de la citerne.

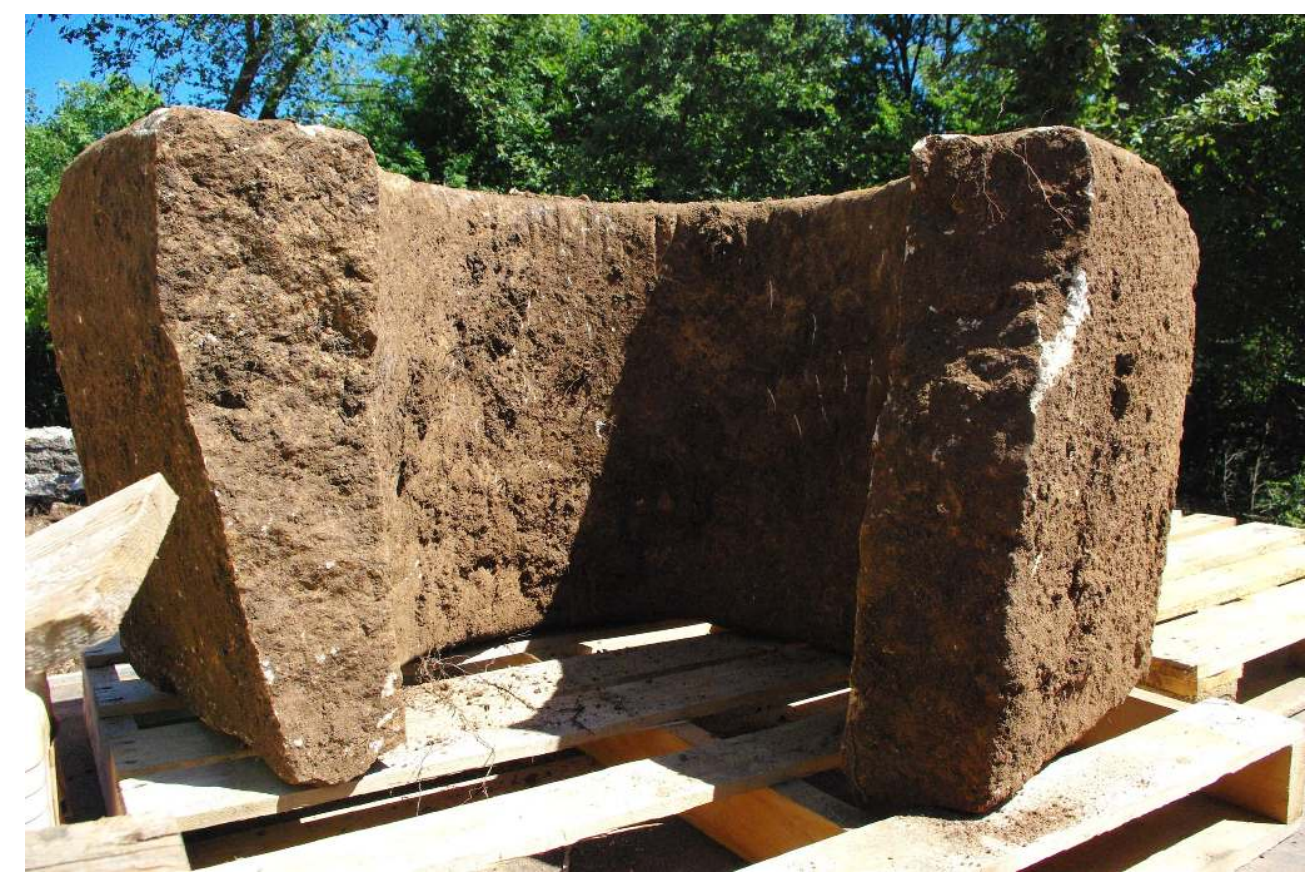

Cl. C. Rousse.

15 Cette citerne est, par ses dimensions, l'une des plus grandes connues en Istrie ${ }^{8}$. Si l'on considère la hauteur conservée des murs, le volume d'eau contenu dépassait les $350 \mathrm{~m}^{3}$. Des départs de murs ont été mis à jour sur le côté ouest de la citerne, en direction de la plage où sont connus d'autres tronçons de murs antiques. On peut donc penser que cette structure hydraulique servait à alimenter un important secteur résidentiel situé en contrebas vers la mer, peut-être la villa maritime des propriétaires de Loron. Du point de vue des techniques de construction, d'étroites similitudes existent entre la citerne de Santa Marina et la grande réserve d'eau déjà fouillée dans l'atelier de Loron ${ }^{9}$, mais aussi avec la citerne antique de Stancija Blek ${ }^{10}$, autre domaine voisin et contemporain de Loron ${ }^{11}$. En tout cas, l'état de conservation remarquable des murs, des enduits et du pavement de la citerne a attiré l'attention des services archéologiques (surintendance) et de la municipalité, dans une perspective de protection et de valorisation. Il justifie de poursuivre l'étude de la citerne et de son environnement immédiat, dans la superficie (terrain public) qui nous est actuellement accessible.

16 Les résultats acquis durant cette campagne de fouille conduisent à développer de façon distincte l'étude du secteur de Santa Marina (2014) et du quartier servile de Loron (2015), en poursuivant parallèlement les prospections diachroniques sur l'ensemble du promontoire: l'objectif d'un relevé LiDAR de la zone est maintenu, de même que le développement des études paléo-environnementales pour mieux comprendre l'évolution du paysage littoral et les dynamiques d'occupation à l'époque antique et post-antique ${ }^{12}$. 


\section{BIBLIOGRAPHIE}

Les comptes-rendus des fouilles sont publiés chaque année dans la revue croate Histia Antiqua et dans les Chroniques des activités archéologiques de l'École française de Rome. Par simplification, seules ces dernières sont citées ici en bibliographie.

Benčić 2006 = G. Benčić, I siti archeologici del territorio di Torre, Fratta ed Abrega, dans D. L. Ratković (dir.), Torre, Fratta, Abrega, patrimonio culturale, Parenzo, 2006 (Heredas Histriae, I), p. 275-298.

Bruzek 1991 = J. Bruzek, Fiabilité des procédés de détermination du sexe à partir de l'os coxal : implications pour l'étude du dimorphisme sexuel de l'Homme fossile, thèse de doctorat de l'Institut de paléontologie humaine, Muséum national d'histoire naturelle, Paris, 1991.

Carre - Kovačić - Tassaux 2011 = M.-B. Carre, V. Kovačić, F. Tassaux, L'Istrie et la mer : la côte du Parentin dans l'Antiquité, Bordeaux, 2011 (Ausonius, Mémoires, 25), trad. croate : Sjeverno priobalje poreštine u antici, Poreč, 2012.

Fiò 2012 = V. Fiò, Il fenomeno della moneta in tomba di epoca medievale nell'Italia nord-occidentale : significati, continuità e rinnovamento di un rito, thèse de doctorat sous la direction de T. M. Lucchelli, Università Ca' Foscari de Venise, 2012.

Loron I = F. Tassaux, R. Matijašić, V. Kovačić, Loron (Croatie) : un grand centre de production d'amphores à huile istriennes (Ir-IV e. p.C.), Bordeaux, 2001 (Ausonius, Mémoires, 6).

Matijašić 1998 = R. Matijašić, Gospodarstvo antičke Istre, Pula, 1998.

Raxter - Auerbach - Ruff $2006=$ M. H. Raxter, B. M. Auerbach, C. B. Ruff, Revision of the Fully technique for estimating statures, dans American Journal of Physical Anthropology, 130, 2006, p. 374-384.

Rousse 2008 = C. Rousse, Il sito di Loron : l'organizzazione del complesso produttivo, dans G. Lipovac Vrkljan et I. Radić Rossi (dir.), officine per la produzione di ceramica e vetro in epoca romana [Atti del convegno, Crikvenica, 23-24 octobre 2008], Rijeka, 2011, p. 75-82.

Rousse - Tassaux 2009 = C. Rousse, F. Tassaux, Loron (Tar-Vabriga, Croatie), dans MEFRA, 121,1, 2009, p. 253-263.

Rousse - Tassaux 2011 = C. Rousse, F. Tassaux, Loron (Tar-Vabriga, Croatie), dans MEFRA, 124, 1, 2001, p. 273-277.

Rousse - Tassaux 2012=C. Rousse, F. Tassaux, Loron (Tar-Vabriga, Croatie), dans Chronique des activités archéologiques de l'École française de Rome [en ligne], 2012, URL: http://cefr.revues.org/217.

Rousse - Carre - Kovačić 2013= C. Rousse, M.-B. Carre, V. Kovačić, Loron / Santa Marina - Busuja (Tar-Vabriga, Poreč, Croatie), dans Chronique des activités archéologiques de l'École française de Rome [en ligne], 2013, URL: http://cefr.revues.org/1009.

Schmitt 2005= A. Schmitt, Une nouvelle méthode pour estimer l'âge au décès des adultes à partir de la surface sacro-pelvienne iliaque, dans Bulletins et mémoires de la Société d'anthropologie de Paris, 17, 1-2, 2005, p. 89-101.

Šilijeg - Kovačić - Konestra 2012= B. Šilijeg, V. Kovačić, A. Konestra, Arheološko-konzervatorska istraživanja lokaliteta Stancija Blek kod Tara u 2011.g. (Archaeological and conservation research of the 
Sancija Blek site near Tar in 2011), dans Annales Instituti Archaeologici - Godišnjak Instituta za arheologiju , VIII, 2012, p. 103-106.

\section{NOTES}

1. Aucun vestige n'a pour l'instant été repéré plus au nord, dans le prolongement de ces édifices. En 2012, des prospections magnétiques ont repérés des anomalies ténues, pouvant indiquer la présence de structures dont l'orientation semble cependant différente du plan du complexe antique (Rousse - Carre - Kovačić 2012). Ces éléments n'ont pas encore été vérifiés sur le terrain.

2. Benčić 2006.

3. Fio 2012.

4. Schmitt 2005.

5. Bruzek 1991.

6. Raxter - Auerbach - Ruff 2006, p. 374-384.

7. Rousse - Tassaux 2011 ; Rousse - Carre - Kovačić 2012.

8. Matijašić 1998.

9. Rousse - Tassaux 2009.

10. Šilijeg - Kovačić - Konestra 2012.

11. Carre - Kovačić - Tassaux 2011.

12. Le projet d'un relevé LiDAR est actuellement en cours de développement, au sein d'un atelier thématique de recherche interdisciplinaire soutenu par le LabexMed (Aix-Marseille université). Il fait également l'objet d'une collaboration avec E. Fovet et le laboratoire Chrono-Environnement de l'université de Franche Comté. Le développement des recherches paléo-environnementales est conduit au sein du programme ISTRIE soutenu par le ministère des Affaires étrangères, sur les sites de Loron et de Busuja.

\section{INDEX}

Mots-clés : villa, atelier, citerne, amphore, monnaie, sépulture, prospection, époque romaine Index géographique : Loron

institutions Zavičajni muzej Poreštine, École française de Rome, Aix-Marseille Université, Centre Camille Jullian

\section{AUTEURS}

\section{CORINNE ROUSSE}

Aix-Marseille Université, CNRS, UMR 7299 « Centre Camille Jullian » - corinne.rousse

[at]gmail.com

\section{VLADIMIR KOVAČIĆ}

Zavičajni musej Poreštine (Poreč, Croatie) - vladimir.kovacic[at]muzejporec.hr 


\section{SOLENN DE LARMINAT}

Centre « Antiquité classique et tardive », UMR 8167 Orient et Méditerranée

\section{VALERIO DE LEONARDIS}

Vrije Universiteit Amsterdam (VU), Università degli studi di Roma Tre

\section{PAOLA MAGGI}

Università degli studi di Trieste

\section{CLAUDIO TAFFETAN}

Aix-Marseille Université, UMR 7299 « Centre Camille Jullian , Università degli studi di Roma Tre

\section{FABRIZIO TERRIZZI}

Università degli studi di Roma « Tor Vergata » 